OPEN ACCESS

Edited by:

Daliang Ning,

University of Oklahoma, United States

Reviewed by:

Xiaoqian Yu,

University of Vienna, Austria

Hui Li,

Institute of Applied Ecology (CAS),

China

*Correspondence:

Zhanshan (Sam) Ma

ma@vandals.uidaho.edu

Specialty section:

This article was submitted to

Systems Microbiology,

a section of the journal

Frontiers in Microbiology

Received: 24 April 2021

Accepted: 15 July 2021

Published: 20 August 2021

Citation:

Ma ZS (2021) Evaluating

the Assembly Dynamics in the Human

Vaginal Microbiomes With

Niche-Neutral Hybrid Modeling.

Front. Microbiol. 12:699939.

doi: 10.3389/fmicb.2021.699939

\section{Evaluating the Assembly Dynamics in the Human Vaginal Microbiomes With Niche-Neutral Hybrid Modeling}

\author{
Zhanshan (Sam) Mat,2* \\ ${ }^{1}$ Computational Biology and Medical Ecology Lab, State Key Laboratory of Genetic Resources and Evolution, Kunming \\ Institute of Zoology, Chinese Academy of Sciences, Kunming, China, ${ }^{2}$ Center for Excellence in Animal Evolution \\ and Genetics, Chinese Academy of Sciences, Kunming, China
}

Using 2,733 longitudinal vaginal microbiome samples (representing local microbial communities) from 79 individuals (representing meta-communities) in the states of healthy, BV (bacterial vaginosis) and pregnancy, we assess and interpret the relative importance of stochastic forces (e.g., stochastic drifts in bacteria demography, and stochastic dispersal) vs. deterministic selection (e.g., host genome, and host physiology) in shaping the dynamics of human vaginal microbiome (HVM) diversity by an integrated analysis with multi-site neutral (MSN) and niche-neutral hybrid (NNH) modeling. It was found that, when the traditional "default" $P$-value $=0.05$ was specified, the neutral drifts were predominant ( $\geq 50 \%$ metacommunities indistinguishable from the MSN prediction), while the niche differentiations were moderate ( $<20 \%$ from the NNH prediction). The study also analyzed two challenging uncertainties in testing the neutral and/or nicheneutral hybrid models, i.e., lack of full model specificity - non-unique fittings of same datasets to multiple models with potentially different mechanistic assumptions - and lack of definite rules for setting the $P$-value thresholds (also noted as $P_{t}$-value when referring to the threshold of $P$-value in this article) in testing null hypothesis (model). Indeed, the two uncertainties can be interdependent, which further complicates the statistical inferences. To deal with the uncertainties, the MSN/NNH test results under a series of $P$-values ranged from 0.05 to 0.95 were presented. Furthermore, the influence of $P$-value threshold-setting on the model specificity, and the effects of woman's health status on the neutrality level of HVM were examined. It was found that with the increase of $P$-value threshold from 0.05 to 0.95 , the overlap (non-unique) fitting of $\mathrm{MSN}$ and $\mathrm{NNH}$ decreased from 29.1 to $1.3 \%$, whereas the specificity (uniquely fitted to data) of MSN model was kept between 55.7 and $82.3 \%$. Also with the rising $P$-value threshold, the difference between healthy and BV groups become significant. These findings suggested that traditional single $P$-value threshold (such as the de facto standard $P$-value $=0.05$ ) might be insufficient for testing the neutral and/or niche neutral hybrid models.

Keywords: unified neutral theory of biodiversity and biogeography, multi-site neutral model, niche-Neutral hybrid model, human vaginal microbiome, bacterial vaginosis, hierarchical Dirichlet process 


\section{INTRODUCTION}

The relationship between human vaginal microbiome (HVM) and women's health has been investigated since the 1980s, when clinical microbiologists had postulated that the diversity and possibly stability of vaginal microbiome are involved in the occurrence/recurrence of bacterial vaginosis (BV) (e.g., Sobel, 1999; Fredricks et al., 2005; Fredricks, 2011; Ma et al., 2012). Those studies are among the earliest ecological approaches to diseases now often referred to as the human MADs (microbiomeassociated diseases) with an ever more rapidly growing list including BV, IBD (inflammatory bowel disease), periodontitis, cystic fibrosis (CF), psoriasis and many others (Lynch and Pedersen, 2016; Knight et al., 2017; Young, 2017; Gilbert et al., 2018). The metagenomics technique and the launch of the human microbiome project (HMP) and MetaHIT (metagenome of human intestinal tract) have revolutionized the investigation of the human microbiome and associated diseases during the last decade or so. Nevertheless, many questions in the field are still open and new more complex questions are being raised. In the case of $\mathrm{BV}$ and vaginal microbiome, as described by Fredricks (2011) who borrowed Winston Churchill's words for a very different topic, " $B V$ remains a riddle, wrapped in a mystery, and inside an enigma." A recent characterization "that $B V$ is not a single entity, but a syndrome linked to various community types that cause somewhat similar physiological symptoms." by Ma et al. (2012) reflects the state-of-the-art understanding of BV etiology. Obviously, although the importance of vaginal microbiome ecology in BV etiology is repeatedly confirmed, the mechanistic relationship between BV and HVM is far from clear. A pair of questions of fundamental importance: what are the underlying mechanisms driving the dynamics of HVM and what are their implications to the occurrence/recurrence of BV, are still largely unanswered.

Addressing the question of community assembly and diversity maintenance, the essential ingredients of community structure and dynamics, has attracted extensive attention and also led to vigorous debate (Alonso et al., 2006; McGill et al., 2006; Chisholm and Pacala, 2010; Rosindell et al., 2011). Two leading and competing theories in this field have been the traditional niche theory with a history back to the 1910s (Grinnell, 1917; Hutchinson, 1957; Holt, 2009) and more recent neutral theory (Hubbell, 2001). Both theories were invented to explain a familiar phenomenon on the earth, which was described by Darwin (1859) in the last paragraph of his "On the Origin of Species" as "It is interesting to contemplate a tangled bank, clothed with many plants of many kinds, with birds singing on the bushes, with various insects flitting about, and with worms crawling through the damp earth, and to reflect that these elaborately constructed forms, so different from each other, and dependent upon each other in so complex a manner, have all been produced by laws acting around us." In modern ecological terminology, entangled bank is essentially the concept of ecological community. Darwin was wondering how diverse lives (species) could coexist and form a beautiful entangled bank, while his theory stipulated the universal struggle for life as a consequence to natural selection. The classic niche theory assumed that each species has its own niche in which its individuals are adapted to live and prosper, and the entangled bank consists of many different niches suitable for many different species. In terms of niche theory, deterministic traits a species possess or selective niche forces play critical roles in driving the assembly of an ecological community as well as the maintenance of diversity after the community is established.

In the late 1990s, Hubbell (2001) challenged the traditional niche view by proposing the unified neutral theory of biodiversity and biogeography (UNTB). Different from traditional niche theory, the UNTB was formulated as a probability distribution model, which can be fitted with the species abundance distribution data (the number of each species in a community), obtainable by sampling ecological communities, and rigorously tested statistically. The theory assumes that the individuals of all species in a community are demographically equivalent, but their birth/death rates are stochastic, which means birthdeath, migration, and speciation are all random events. Consequently, random drift and dispersal play critical roles in driving community assembly and diversity maintenance. Some researchers argued that the concept of species equivalence is "flawed" given the existence of niche differences and competitive asymmetries among species. Nevertheless, the stochasticity in species demography (particularly of single-cell microbes) is also a biological reality and its role may not be ignored in many communities. In reality, both deterministic niche forces and stochastic neutral forces may be in effect in setting the rules of community assembly and diversity maintenance, and it may be the hybrid effects that shape the community dynamics. For this reason, in the last decade and so, several hybrid models that integrate neutral and niche effects have been developed (e.g., Tilman, 2004; Ofiteru et al., 2010; Stokes and Archer, 2010; Jeraldo et al., 2012; Pigolotti and Cencini, 2013; Tang and Zhou, 2013; Fisher and Mehta, 2014; Kalyuzhny et al., 2014a,b, 2015; Matthews and Whittaker, 2014; Noble and Fagan, 2015). As to the debates on the usefulness and validity of the UNTB, using an analogy, in modern statistics (especially in biostatistics), it has been widely recognized that many datasets do not follow the Gaussian distribution (the normal distribution); nevertheless, few statisticians would question the foundational role of the Gaussian distribution, not to mention its validity. Similarly, the merits and unique advantage of UNTB as a null model for testing the significance of stochastic drift and dispersal have been firmly established and widely applied in the community ecology of plants and animals.

In the present study, we use a pair of models, the first a multi-site neutral model (Harris et al., 2017) and the second, a niche-neutral hybrid model (Tang and Zhou, 2013), to evaluate the relative significance of neutral and niche effects in shaping the dynamics of HVM. We further investigate the difference in the neutral-niche continuum between BV patients and healthy women. Our approach is different from most existing applications of neutral or hybrid models in the following three aspects.

First, most existing neutral or niche-neutral hybrid models use spatially implicit/explicit community/metacommunity data, whereas we use longitudinal (time-series) sampling of the 
community/metacommunity. In spatially explicit models, the metacommunity consists of multiple local communities, which are connected with each other through dispersal and migration. In temporal (time-series) models, the metacommunity consists of a series of "snapshots" of the same community at different time points, i.e., the time-series data obtained from sampling the vaginal microbiome of a subject at different time points in this study. Indeed, previously, Kalyuzhny et al. (2014a,b, 2015) used time-series data to perform dynamic analysis of the niches versus neutrality and they termed the analysis as a generalized neutral theory for explaining the static and dynamic properties of ecological communities. A reason we did not adopt their models is that the models we use in this study, as explained below, are truly multi-site mechanistically, which are mapped to the time-series points in our study.

Second, we use a truly multi-site neutral (MSN) model of UNTB, which was developed by Harris et al. (2017) to overcome the severe computational limitation of existing neutral theory models when the number of local communities is large and the migration rates among the local communities are different (Etienne, 2007, 2009a,b). The core technique Harris et al. (2017) developed was to approximate the multi-site UNTB model with the hierarchical Dirichlet process (HDP) and use an efficient Bayesian machine-learning algorithm. With their approach, fitting even the largest dataset can be performed in a reasonable amount of time. This important computational advance enables us to build a UNTB model for each subject by utilizing the time series sampling of her vaginal microbiome. This capability is of significant practical importance given the established connection between BV and the diversity of the vaginal microbiome, in particular, a long-standing puzzle in $\mathrm{BV}$ etiology - the rise of species diversity associated with BV (e.g., Sobel, 1999; Fredricks et al., 2005; Fredricks, 2011; Ma et al., 2012; Ma and Ellison, 2018, 2019).

Third, we also apply the niche-neutral hybrid (NNH) model by Tang and Zhou (2013) to further assess the neutral-niche hybrid effects in shaping the dynamics of HVM diversity. A major reason we prefer this hybrid model to other existing hybrid models (e.g., Tilman, 2004; Ofiteru et al., 2010; Stokes and Archer, 2010; Jeraldo et al., 2012; Pigolotti and Cencini, 2013; Tang and Zhou, 2013; Fisher and Mehta, 2014; Kalyuzhny et al., 2014a,b, 2015; Matthews and Whittaker, 2014; Noble and Fagan, 2015) is because both MSN and NNH use exactly the same data collection methods - either multi-site or multi-time-point sampling. The only essential difference between the neutral-niche hybrid model (NNH) and multi-site neutral model (MSN) is the assumption that niche differences exist among local communities in $\mathrm{NNH}$, while the MSN assumes no niche differentiation. In a timeseries setting, the NNH model can tell us whether deterministic forces (similar to habitat selection in a spatial setting) such as when changes in the host's physiology significantly influence the dynamics of vaginal microbiome diversity over time.

In summary, by building and testing the MSN and $\mathrm{NNH}$ models for each subject, we are able to evaluate the relative importance of stochastic forces (e.g., neutral dispersal, drift, and stochastic diversification) vs. deterministic forces (e.g., microbial interactions, host genome and physiology, menses, etc.) in shaping the dynamics of community diversity. Furthermore, if we treat BV or health status as part of the host physiology, testing the MSN/NNH models can reveal the impact of BV on the dynamics of the HVM diversity (assuming that diversity change is the consequence of $\mathrm{BV}$ ), or reveal the diversity changes that induce BV (assuming that diversity change is the cause of $B V)$. Regardless of the causal assumption, our approach offers a useful tool for evaluating the mechanisms (niche vs. neutral) of the dynamics of HVM diversity as well as the factors affecting the balances between different mechanisms. We demonstrate our approach (see Figure 1) by using the datasets (see Table 1) from three separate longitudinal studies on the HVM, including 79 subjects sampled at 2,733 time points (Gajer et al., 2012; Ravel et al., 2013; Romero et al., 2014).

\section{MATERIALS AND METHODS}

\section{Human Vaginal Microbiome (HVM) Datasets and Analysis Strategy}

Table 1 below listed the three published datasets (groups) of the HVM (human vaginal microbiome), which are reanalyzed in this study to perform the niche-neutral theoretic analysis. Figure $\mathbf{1}$ is a diagram illustrating the background, objectives and the integrated niche-neutral approach to achieving the objectives of this study outlined previously. Two mathematical models: the multi-site neutral model (MSN) by Harris et al. (2017) and niche-neutral hybrid model (NNH) by Tang and Zhou (2013), are used to fit the same HVM datasets. Both the models are extensions or derived from Hubbell (2001) unified neutral theory of biodiversity and biogeography (UNTB). Brief description of the MSN and NNH models as well as their fittings is presented in Table 1. It is noted that these datasets are from three independent studies: minor difference in sequencing protocols may exist. For this reason, the samples from each of the 79 individuals are modeled independently. With the independent modeling, the influence from sequencing protocols should have minimized.

\section{The Multi-Site Neutral Model (MSN) by Harris et al. (2017) Hubbell's Unified Neutral Theory of Biodiversity (UNTB)}

The UNTB conceptually distinguishes between metacommunity dynamics from local community dynamics coupled to metacommunity through migrations. The theory assumes that both the dynamics are driven by similar neutral processes, except that in metacommunity speciation, rather than migration are in operations (Hubbell, 2001, 2006). The neutral process or ecological equivalence between species implies that the demographic rates (birth/death) of all species are stochastic but equivalent on per capita basis (Harris et al., 2017). There are three key parameters (elements) with the UNTB, the immigration rate $\left(I_{i}\right)$, which controls the coupling of a local community to the metacommunity. Another is the speciation rate, also known as the fundamental biodiversity number $(\theta)$, which can be interpreted as the rate at which new individuals are added to the 


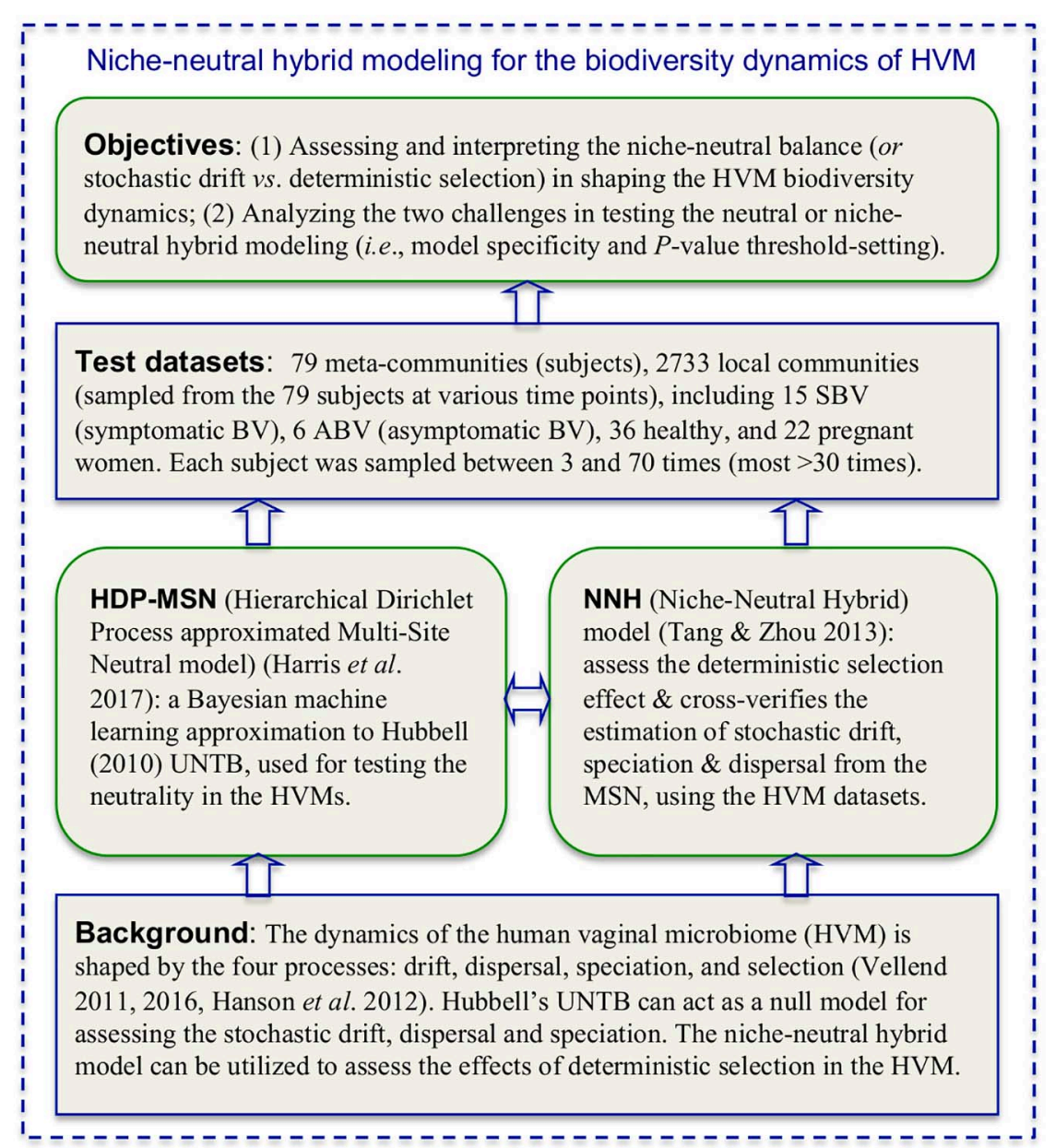

FIGURE 1 | A diagram illustrating the background, objectives and the integrated niche-neutral theoretic-approach to achieving the objectives of this study based on 2,733 longitudinal metagenomics (16S-rRNA) samples collected from 79 women (including BV patients).

TABLE 1 | The datasets of multi-site HVM (human vaginal microbiome) datasets utilized for testing the MSN (multi-site neutral) and NNH (niche-neutral hybrid) models.

\begin{tabular}{|c|c|c|c|c|}
\hline Datasets & ${ }^{*} N$ & $* * S$ & Sample description & Sources \\
\hline HEA-1 (Healthy 1) & 4 & $66 \sim 69$ & $\begin{array}{l}\text { Ravel et al. (2013) sampled and DNA-pyrosequenced the vaginal microbiota of } \\
\text { a cohort of } 25 \text { subjects over a } 10 \text {-week period, consisting of } 15 \text { SBV, } 6 \text { ABV, } \\
\text { and } 4 \text { healthy subjects (HEA-1). Total } 16 \mathrm{~S}-\text {-rRNA reads }=8,757,681 \text {, Average } \\
\text { reads }=5285 \text {, The dataset is available from: } \\
\text { https://doi.org/10.1186/2049-2618-1-2 }\end{array}$ & $\begin{array}{l}\text { Ravel et al. (2013) } \\
\text { Microbiome }\end{array}$ \\
\hline $\begin{array}{l}\text { HEA-2 (Healthy 2): "32-healthy" cohort } \\
\text { of HVMC study }\end{array}$ & 32 & $25 \sim 33$ & $\begin{array}{l}\text { Gajer et al. (2012) sampled and DNA-pyrosequenced the vaginal microbiota of } \\
\text { a cohort of } 32 \text { healthy individuals (HEA-2). Total } 16 S-r R N A \text { reads }=2,522,080 \\
\text { from } 937 \text { samples; Average reads }=2692 \text {. The OTU table is available at: } \\
\text { doi: } 10.1126 / \text { scitranslmed. } 3003605\end{array}$ & $\begin{array}{l}\text { Gajer et al. (2012) } \\
\text { Science Translational } \\
\text { Medicine }\end{array}$ \\
\hline PREG (Pregnancy) & 22 & $3 \sim 8$ & $\begin{array}{l}\text { The vaginal microbiomes of a cohort of } 22 \text { normally pregnant women were } \\
\text { sampled } 6 \text { times (for each individual) and DNA pyrosequenced. Total 16S-rRNA } \\
\text { reads = 567,448; Average reads = 4082; The dataset is available at: } \\
\text { https://doi.org/10.1186/2049-2618-2-4 }\end{array}$ & $\begin{array}{l}\text { Romero et al. (2014) } \\
\text { Microbiome }\end{array}$ \\
\hline Total or range & 79 & $3 \sim 70$ & $\begin{array}{l}\text { A total of } 79 \text { meta-communities and 2,733 local communities (time-series samples) } \\
\text { conduct the tests. }\end{array}$ & \\
\hline
\end{tabular}

${ }^{*} N=$ the number of subjects (individuals) included in each dataset. ${ }^{*} \mathrm{~S}=$ the approximate number of time points when samples of the HVMC were taken from each individual. 
metacommunity due to speciation. The third aspect of the UNTB is to assume that the SAD (species abundance distribution) of each community sample can be described by the multinomial (MN) distribution, formally:

$$
\overline{X_{i}} \sim \operatorname{MN}\left(N_{i}, \bar{\pi}_{i}\right)
$$

where $N_{i}$ is the size of $i$-th local community, $\bar{\pi}_{i}$ is a vector of the probability of observing a particularly species at $i$-th local community (Harris et al., 2017).

\section{UNTB-HDP (Hierarchical Dirichlet Process) Limit to Metacommunities}

A fully general case of fitting multiple sites (local communities) UNTB with potentially different immigration rates is computationally extremely challenging (actually intractable) even for small number of sites, and approximate algorithms must be utilized (Harris et al., 2017). Harris et al. (2017) developed an efficient Bayesian fitting framework by approximating the neutral models with the hierarchical Dirichlet process (HDP). The approximation was able to encapsulate the three essential elements of Hubbell (2001) UNTB, as stated above, but offers an efficient Bayesian fitting strategy for the multi-site UNTB.

Sloan et al. $(2006,2007)$ showed that for large local population sizes, assuming a fixed finite-dimensional metacommunity distribution with $S$ species present, the local community distribution, $\bar{\pi}$, can be approximated by a Dirichlet distribution (Sloan et al., 2006, 2007). But it was Harris et al. (2017) who developed the general framework for approximating the UNTB computationally efficiently. Assuming there is a potentially infinite number of species that can be observed in the local community, then the stationary distribution of observing local population $i$ is a Dirichlet process (DP), i.e.,

$$
\bar{\pi}_{i} \mid I_{i}, \bar{\beta} \sim D P\left(I_{i}, \bar{\beta}\right)
$$

where $\bar{\beta}=\left(\beta_{1}, \ldots, \beta_{S}\right)$ is the relative frequency of each species in the metacommunity, and $I_{i}$ is the immigration rate.

At the metacommunity level, a Dirichlet process can still be used, but the base distribution is simply a uniform distribution over arbitrary species labels. The metacommunity distribution is then a purely stick breaking process, i.e.,

$$
\bar{\beta} \sim \operatorname{Stick}(\theta)
$$

where $\theta$ is the fundamental biodiversity number. $\theta$ is a function of speciation rate $(s)$ in the form of $\theta=(s /(1-$ $s)(N-1)$, where $N$ is the size of metacommunity (i.e., the fixed number of individuals in the metacommunity). The total number of species $(S)$ in the metacommunity proportionally increases with $\theta$. In addition, when $\theta$ increases, the SAD (species abundance distribution) is increasingly skewed to low abundance rare species (Harris et al., 2017). Note that speciation in the metacommunity is a counterpart of migration in a local community, except that the speciation is in operation on a longer timescale than migration. For this reason, both immigration rate $\left(I_{i}\right)$ and biodiversity number $\theta$ have similar structure in their models. Specifically, $I_{i}=\left(m_{i} /\left(1-m_{i}\right)\left(N_{i}-\right.\right.$ 1 ), where $m_{i}$ is the immigration probability to local community $i$, and $N_{i}$ is the local community size. Obviously, when $I_{i} \rightarrow \propto$, the stationary distribution of local community should approach the metacommunity distribution since that means migration probability is equal to 1 , i.e., all members in the local community are immigrants. When $I_{i} \rightarrow 0$, local community can become dominated by a single species (Harris et al., 2017).

Given that both local community and metacommunity are approximated with Dirichlet processes, the problem can be formulated as a hierarchical Dirichlet process (HDP) (Teh et al., 2006; Harris et al., 2017). Alternatively, Dirichlet process (DP) can also be formulated as the so-called Chinese restaurant process, from which the Antoniak equation can be derived. The Antoniak equation represents the number species $(S)$ observed following $N$ draws from a Dirichlet process with biodiversity number $\theta$, and is with the following form:

$$
P(S \mid \theta, N)=s(N, S) \theta^{S} \frac{\Gamma(\theta)}{\Gamma(\theta+N)}
$$

where $s(N, S)$ is the unsigned Stirling number of the first kind and $\Gamma$ (.) denotes the gamma function (Antoniak, 1974).

\section{Gibbs Sampler (MCMC Algorithm) for the UNTB-HDP Model}

The full UNTB-HDP model is obtained by combining previous equations (1-3) and also the distribution models of biodiversity number $(\theta)$ and immigration rate $\left(I_{i}\right)$, both of which are assumed to follow Gamma distribution. Harris et al. (2017) developed an efficient Gibbs sampler for the UNTB-HDP approximation, which is a type of Bayesian Markov Chain Monte Carlo (MCMC) algorithm and can be summarized as the following four sampling steps, including sampling the biodiversity parameter, sampling the metacommunity distribution, sampling the immigration rate, and sampling the ancestral states. Harris et al. (2017) found through experiments that to ensure sampling was performed with the stationary distribution, 50,000 Gibb samples for each fitted dataset were necessary with the first 25,000 iterations removed as burn-in. The results are reported as the median values over the last 25,000 samples with upper and lower credible limits (Bayesian confidence) given by 2.5 and $97.5 \%$ quantiles of those samples.

\section{Fitness Tests for the UNTB-HDP Multi-Site Neutral (MSN) Model}

To determine whether an observed dataset fits the UNTB-HDP multi-site neutral (MSN) model (hereafter shortened as MSN model), Harris et al. (2017) proposed a similar Monte Carlo significance test to that used by Etienne (2007). Furthermore, Harris et al. (2017) also developed a procedure to test for the local neutral community assembly but with a fitted possibly non-neutral metacommunity because of the hierarchical nature of the MSN model. Specifically, with Harris et al. (2017) MSN model, two-level tests (local community and metacommunity levels) for neutrality can be performed. For both the tests, samples were generated from $N=2,500$ sets of fitted MSN parameters, which were selected from every tenth iteration of the last 25,000 Gibbs samples (a total of 50,000 samples were simulated, and the first 25,000 samples were discarded as burn-in). $N=2,500$ 
is chosen to compute the pseudo $P$-values for conducting the neutrality test (Harris et al., 2017). In addition, for each observed community sample, there is the actual log-likelihood $L_{0}$. Two additional parameters $\theta$ and $M$ are particular worthy of mentioning: $\theta$ is the median of the fundamental biodiversity parameters computed from 25,000 times of simulations, and $M$-value is the average of the medians of the migration rates of local communities in each metacommunity, also computed from 25,000 times of simulations.

To test the neutrality at the metacommunity level, $P_{M}$, which is "the proportion of the simulated neutral samples with their likelihoods not exceeding the observed data likelihood" (Harris et al., 2017). The computation of $P_{M}$ is as follows: Assume $L_{M}$ is the median of the log-likelihoods of the simulated neutral metacommunity samples, and $N_{M}$ is the number of simulated neutral metacommunity samples, having their log-likelihoods satisfying $L \leq L_{0}$ (where $L$ is the simulated likelihood and $L_{0}$ is the actual likelihood as mentioned previously), then the $P_{M}=N_{M /} N$ is a pseudo $P$-value for testing the neutrality at metacommunity level. If $P_{M}>0.05$, the metacommunity appears to satisfy the MSN model, according to Harris et al. (2017).

To test the neutrality at the local community level, $P_{L}$, which is the proportion of the simulated locally neutral samples exceeding the observed data likelihood (Harris et al., 2017). It is computed as follows:

Assume $L_{L}$ is the median of the log-likelihoods of the simulated local community samples, and $N_{L}$ is the number of simulated local community samples, having their likelihoods not exceeding the $L_{0}$, then $P_{L}=N_{L} N$, is the pseudo $P$-value for testing the neutrality at the local community level. If $P_{L}>0.05$, the local community appears to satisfy the neutral model. Readers are referred to Harris et al. (2017) for the detailed algorithm and computational procedures (including the software in C language) for fitting the MSN model, which we used for analyzing HVM datasets in this study.

\section{The Niche-Neutral Hybrid (NNH) Model by Tang and Zhou (2013)}

Tang and Zhou (2013) proposed a hybrid niche-neutral model by revising Volkov et al. (2007) neutral model for multiple discrete communities. Volkov et al. (2007) assumed that the inter-species interactions in a steady-state community may be ignored, and all species in the community become functionally equivalent. They further assumed that birth and death probabilities of a species with $n$ individuals are $b_{n}=b(n+\gamma)$ and $d_{n}=d n$, respectively, where $b$ and $d$ are the per-capita density-independent birth and death rates, and $\gamma$ is a parameter for immigration. The migration was assumed to be species-independent, corresponding to immigration from a time-averaged metacommunity in a speciessymmetric manner. This treatment of migration, in effect, ignored any immigration between local communities within the metacommunity, and also, the rates of immigration considered were small. By solving the master equation for the dynamics of a species, Volkov et al. (2007) obtained the probability that a species has $n$ individuals, which follows the negative binomial distribution:

$$
p(n)=\frac{(1-x)^{\gamma}}{\Gamma(\gamma)} \frac{x^{n}}{n !} \Gamma(n+\gamma)
$$

where $x$ is the ratio of the per capita birth to death rate (i.e., $b / d$, a measure of the lifetime reproductive success), and $\Gamma(z)=$ $\int_{0}^{\infty} t^{z-1} e^{-t} d t$, which is equal to $(z-1)$ ! for integer $z$. They further obtained the mean number of species with abundance $n$ :

$$
<\varphi_{n}>=\theta \frac{x^{n}}{n !} \Gamma(n+\gamma)
$$

where $\theta$ is the fundamental biodiversity parameter, and $S$ is the number of observed species.

Tang and Zhou assumed that a semi-isolated local community consists of $K$ non-overlapping niches. Within each niche, a number of species follow their own neutral rules independent of the other K-1 niches. By applying Volkov et al. (2007) neutral model for multiple discrete communities to a single niche of the community, Tang and Zhou (2013) derived the expected number of species with abundance $n$ in niche $i$ as:

$$
<\varphi_{n, i}>=\theta_{i} \frac{x_{i}^{n}}{n !} \Gamma\left(n+\gamma_{i}\right)
$$

where $\theta_{i}$ is the biodiversity parameter for niche $i, x_{i}$ is the ratio of per capita birth to death rates of each species in niche $i$, and $\gamma_{i}$ is a parameters for immigration of niche $i$. The total expected number of species with abundance $n$ in the community consisting of $K$ niches is represented by the following equation:

$$
<\varphi_{n} ; K>=\sum_{i=1}^{K}<\varphi_{n, i}>
$$

Note that Eq. 8 is a summation of Eq. 7 across $K$ niches, i.e., summing up all species with an abundance of $n$ across all $K$ niches. The following Chi-squared test statistic is utilized to determine the goodness-of-fitting for the niche-neutral hybrid model, i.e.,

$$
\chi^{2}=\sum_{n} \frac{\left(E_{n}-O_{n}\right)^{2}}{E_{n}}
$$

where $E_{n}$ is the expected number of species with abundance $n, O_{n}$ is the observed number of species with abundance $n$.

To test the niche-neutral hybrid effects with Tang and Zhou (2013) NNH model, we computed the following items (listed in Supplementary Table 2 of the online supplementary information (OSI) and partially in Table 3), including: the average number of individuals per niche (local community) in each metacommunity $(J)$, the average species numbers per niche (local community) in each metacommunity $(S)$, the average fundamental biodiversity parameter per niche (local community) in each metacommunity $(\theta)$, the average of the migration coefficients $(m)$, the average of the birth to death ratio $(x)$, the average of the migration rate $(\gamma)$. To conduct the $\chi^{2}$-test at the meta-community level, we computed $\chi^{2}$-value [Eq. 9] and associated $P$-value. To test the neutrality at a local community level, Volkov et al. (2003, 2007) approach for fitting the relative species abundance (RSA) 
distribution to their neutral model is adopted. Specifically, we computed and reported (see the last two columns in Supplementary Table 2 and Table 3 ) the number and percentage of local communities (niches) that passed the local neutrality test.

The $P$-value of the Chi-squared test is then used to determine whether or not Tang and Zhou (2013) hybrid model is suitable for a series of microbial communities sampled from each individual. In the case of our time-series microbiome datasets, we treat each time point as a niche occupied by a local microbial community and fit the neutral model for each local community. Specifically, at the metacommunity level, if $P$-value $>0.05$, then the metacommunity appears to satisfy the $\mathrm{NNH}$, and the metacommunity assembly is co-driven by both niche and neutral processes, which also implies that the metacommunity itself does not satisfy the neutral theory, but within each niche, the local community is neutral. If $P$-value $<0.05$, the metacommunity does not seem to satisfy the $\mathrm{NNH}$, which also implies that within each niche, the local community is not neutral either, and the metacommunity assembly is solely influenced by the niche process. Readers are referred to Tang and Zhou (2013) for the detailed algorithm and computational procedures (including the software) for fitting the NNH model, which we used for analyzing HVM datasets in this study.

\section{Model Specificity and $\boldsymbol{P}$-Value Threshold Setting in Testing the Null Models}

Two uncertainties have been well recognized in testing the neutral theory and niche-neutral hybrid models including the previous MSN and NNH models. One is the lack of full model specificity in fitting the neutral and/or niche-neutral hybrid models such as MSN/HHH models, and another is the lack of definite rules for setting the $P$-value thresholds in testing null models. What makes the statistical inferences more difficult is the potential interdependence between both uncertainties. There are no silver bullets to resolve them for various reasons including the complexity of the problem per se and limitations of the $P$-value setting in frequentist approaches to statistical inferences. In this article, no perfect solutions are offered, but we present two measures to relieve both issues. First, to evaluate the specificity of the MSN/NNH models, we classify the model-fittings as four possible categories: MSNonly, NNH only, both MSN \& NNH, neither MSN nor MSN, and further observe the change of category proportions when $P$-value thresholds were specified differently. This allows us, at the minimum, to have an educated guess for the specificity of each model, particularly under different confidence levels $(P$ values). Second, besides testing the null models (MSN/NNH) under the traditional "default" $P$-value $=0.05$, the results of model testing under a series of $P$-value thresholds are presented and analyzed. The variable $P$-value thresholds allow us to assess the goodness-of-fitting of the MSN/NNH models under various levels of confidence.

\section{RESULTS}

\section{The Niche-Neutral Continuum in Shaping the HVM Dynamics Evaluated Under Traditional "Default" $P$-Value Threshold}

Supplementary Table 1 in the OSI listed the full test results for the MSN with five HVM (human vaginal microbiome) datasets (groups) outlined in Table 1. Table 2 below was excerpted from Supplementary Table 1 to exhibit the results of 9 selected metacommunities. Similarly, Table 3 below exhibited the results of

TABLE 2 | Fitting the HDP-MSN (hierarchical Dirichlet process approximated multi-site neutral model) (Harris et al., 2017) to the HVM (human vaginal microbiome) datasets for selected individuals, excerpted from Supplementary Table 1 in the OSI*.

\begin{tabular}{|c|c|c|c|c|c|c|c|c|c|c|c|c|}
\hline \multirow[t]{2}{*}{ Datasets } & \multirow[t]{2}{*}{ Case No. } & \multirow[t]{2}{*}{$\mathrm{L}_{0}$} & \multirow[t]{2}{*}{$\theta$} & \multirow[t]{2}{*}{ M-Value } & \multicolumn{4}{|c|}{ Meta-Community } & \multicolumn{4}{|c|}{ Local Community } \\
\hline & & & & & $\mathrm{L}_{M}$ & $\mathbf{N}_{M}$ & $\mathbf{N}$ & $\mathbf{P}_{M}$ & $\mathbf{L}_{L}$ & $\mathrm{~N}_{L}$ & $\mathbf{N}$ & $\mathbf{P}_{L}$ \\
\hline ABV & S12 & -7164.578 & 17.818 & 11.849 & -8855.097 & 2437 & 2500 & 0.975 & -7387.665 & 2158 & 2500 & 0.863 \\
\hline \multirow[t]{3}{*}{ SBV } & S5 & -5929.666 & 15.552 & 7.295 & -7861.124 & 2469 & 2500 & 0.988 & -6220.505 & 2339 & 2500 & 0.936 \\
\hline & S3 & -1749.538 & 4.759 & 5.645 & -1945.254 & 1715 & 2500 & 0.686 & -1781.817 & 1672 & 2500 & 0.669 \\
\hline & $\mathrm{S} 17$ & -10498.771 & 8.166 & 920.730 & -6555.105 & 0 & 2500 & 0.000 & -2900.912 & 0 & 2500 & 0.000 \\
\hline Healthy-1 & S7 & -7330.538 & 12.554 & 17.957 & -10193.354 & 2475 & 2500 & 0.990 & -7530.997 & 2096 & 2500 & 0.838 \\
\hline \multirow[t]{2}{*}{ Healthy-2 } & \#400 & -1609.574 & 12.332 & 9.409 & -3248.037 & 2500 & 2500 & 1.000 & -1766.803 & 2330 & 2500 & 0.932 \\
\hline & \#401 & -3002.396 & 23.955 & 6.088 & -4053.368 & 2495 & 2500 & 0.998 & -3362.560 & 2492 & 2500 & 0.997 \\
\hline \multirow[t]{2}{*}{ Pregnancy } & N002 & -325.419 & 12.392 & 2.623 & -447.710 & 2384 & 2500 & 0.954 & -403.615 & 2378 & 2500 & 0.951 \\
\hline & N003 & -581.146 & 13.098 & 7.923 & -687.729 & 2099 & 2500 & 0.840 & -648.312 & 2244 & 2500 & 0.898 \\
\hline
\end{tabular}

${ }^{*} N=2,500$ is the number of Gibb samples selected from 25,000 simulated communities (i.e., every tenth iteration of the last 25,000 Gibbs samples, a total of 50,000 simulations were performed and with the first 25,000 discarded as burn-in), and the $N$ is used to compute the pseudo P-value below for conducting the neutrality test.

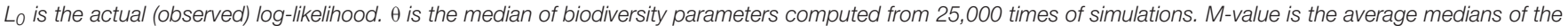

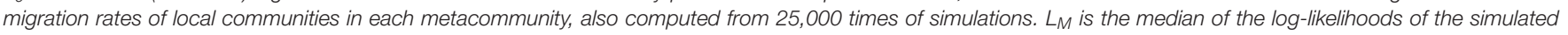

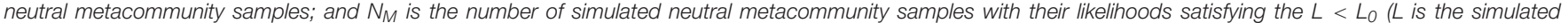

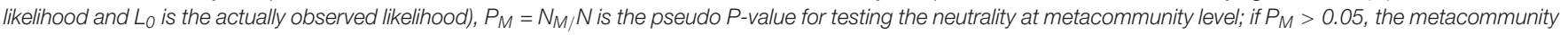
is indistinguishable from the prediction of the MSN model. $L_{L}$ is the median of the log-likelihoods of the simulated local community samples, and $N_{L}$ is the number of

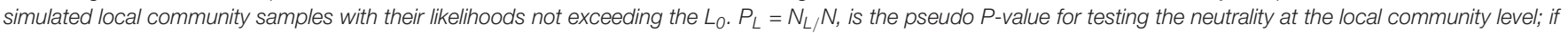
$P_{L}>0.05$, the local community is indistinguishable from the neutral model. See Figure 2 for an example of successfully fitting to the MSN model. 


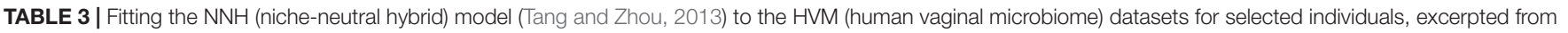
Supplementary Table 2 in the OSI*.

\begin{tabular}{|c|c|c|c|c|c|c|c|c|c|c|c|c|}
\hline Datasets & ID & $J$ & $s$ & $\theta$ & $m$ & $x$ & $\gamma$ & $R^{2}$ & $x^{2}$ & $P$-value & $N^{\text {pass }}$ & $\%$ (pass) \\
\hline $\mathrm{ABV}$ & S12 & 5261.723 & 23.043 & 9277.776 & 0.000 & 0.691 & 0.488 & 0.996 & 52.253 & 0.000 & 22 & 46.8 \\
\hline \multirow[t]{3}{*}{ SBV } & S5 & 4647.896 & 18.042 & 466.265 & 0.001 & 0.643 & 0.557 & 0.983 & 154.291 & 0.000 & 18 & 37.5 \\
\hline & S3 & 164.000 & 8.455 & 4.032 & 0.007 & 0.803 & 1.236 & 0.981 & 0.925 & 0.996 & 11 & 100.0 \\
\hline & $\mathrm{S} 17$ & 5852.400 & 23.940 & 571.207 & 0.000 & 0.724 & 0.531 & 0.986 & 74.385 & 0.000 & 33 & 66.0 \\
\hline Healthy-1 & S7 & 5902.193 & 20.421 & 650.047 & 0.000 & 0.687 & 0.462 & 0.986 & 87.923 & 0.000 & 13 & 37.1 \\
\hline \multirow[t]{2}{*}{ Healthy-2 } & $\# 400$ & 2737.111 & 14.444 & 6.379 & 0.001 & 0.688 & 1.730 & 0.944 & 23.091 & 0.027 & 3 & 33.3 \\
\hline & \#401 & 2614.200 & 22.933 & 7.292 & 0.000 & 0.760 & 1.242 & 0.983 & 12.745 & 0.310 & 13 & 86.7 \\
\hline \multirow[t]{2}{*}{ Pregnancy } & N002 & 4278.000 & 14.500 & 24.256 & 0.000 & 0.541 & 0.890 & 0.974 & 209327 & 0.000 & 1 & 50.0 \\
\hline & N003 & 4215.500 & 22.500 & 3.432 & 0.000 & 0.762 & 1.973 & 0.851 & 5.358 & 0.913 & 4 & 100.0 \\
\hline
\end{tabular}

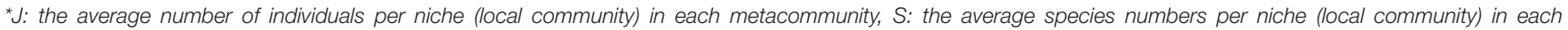

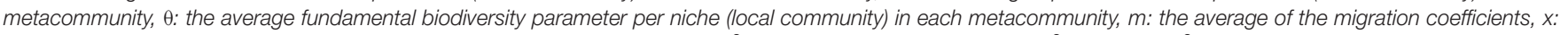

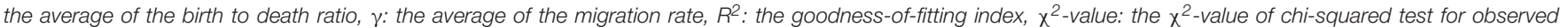

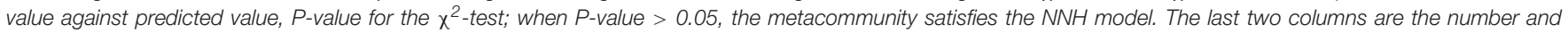
percentage of local communities (niches) that passed the local neutrality test. Note that $R^{2}=1$ resulted from approximation with four effective digits only (e.g., 0.99995,

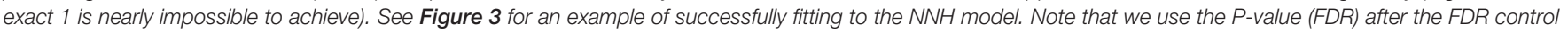
was imposed to determine the outcome of testing the NNH model.

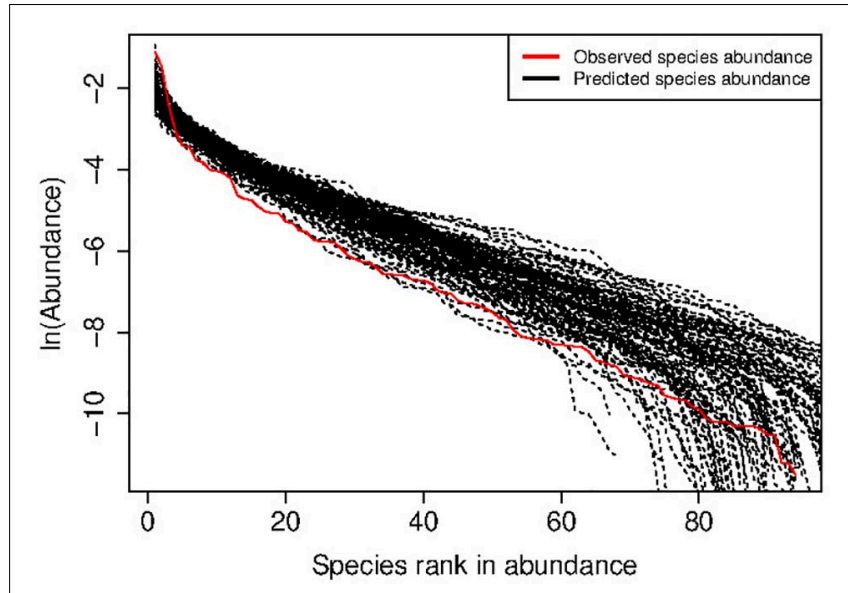

FIGURE 2 | An example of human vaginal microbial metacommunity (Subject\#s35: disease status = SBV) showing successful fitting to the MSN (multi-site neutral) model: the observed species (relative) abundance is estimated from 68 sampling times of the HVM of the subject, and the predicted species abundance is from 25,000 times of MSN simulations.

9 selected meta-communities from Supplementary Table 2 in the OSI, where the full results for fitting the $\mathrm{NNH}$ model were listed. Figures 2, 3 illustrated two examples of fitting the MSN and $\mathrm{NNH}$, respectively.

To better illustrate the full results in Supplementary Tables 1, 2 with Tables 2, 3, we selected 4 meta-communities from each of the five HVM datasets, corresponding to the 4 possible outcomes of testing the MSN and NNH simultaneously (i.e., passing $\mathrm{MSN}$ or $\mathrm{NNH}$ alone, passing both or passing neither). With this scheme, a maximal number of $20(4 \times 5)$ samples could be selected, and it turned out that 11 of the combinations were missing from the results, leading to only 9 meta-communities being selected in Tables 2, 3, respectively. The table legends were noted at the bottom sections of Tables 2, 3 below. Tables 2, 3, therefore, offer windows to inspect the

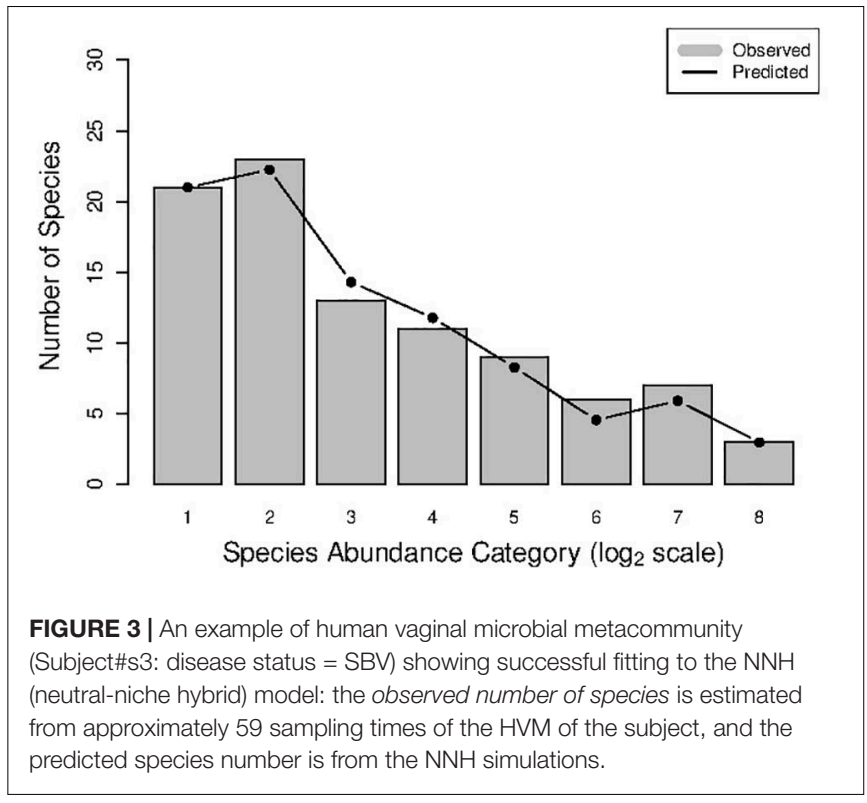

parameters and infer findings from fitting the MSN/NNH models. To inspect the complete test results of the 79 metacommunities and 2,733 local communities, readers are referred to Supplementary Tables 1, 2 in the OSI.

We now try to draw a big picture from the test results (Supplementary Tables 1, 2 and Tables 2, 3) by computing the statistics of the passing rates from testing the MSN and NNH models. Recall that they use exactly the same data formats, i.e., with exactly the same specification for the local community and metacommunity. For example, with the dataset of "32-healthy" cohort, 32 subjects represented 32 metacommunities, and each metacommunity contained 25-33 local communities (or 25-33 niches in the case of $\mathrm{NNH}$ ) given that each subject was sampled 25-33 times. Table 4 (also see Figure 4) below exhibited the passing rates for both MSN 
TABLE 4 | The passing percentages for testing the MSN (multi-site neutral) and $\mathrm{NNH}$ (niche-neutral hybrid) models with the HVM datasets, summarized from Supplementary Tables 1, 2 and a series of the $P$-value thresholds $\left(P_{t}\right)$ for testing MSN/NNH were set to $P_{t}=0.05,0.5,0.9$ or 0.95 .

\begin{tabular}{|c|c|c|c|c|c|c|c|c|}
\hline \multirow[t]{2}{*}{ Microbiome ${ }^{*} N$} & \multicolumn{4}{|c|}{ Meta community } & \multicolumn{4}{|c|}{ Local community } \\
\hline & 0.05 & 0.5 & 0.9 & 0.95 & 0.05 & 0.5 & 0.9 & 0.95 \\
\hline
\end{tabular}

\begin{tabular}{lccccccccc}
\hline The passing percentage (\%) & of MSN (Multi-site neutral) model \\
ABV & 6 & 100 & 67.7 & 33.3 & 16.7 & 100 & 50 & 0 & 0 \\
SBV & 15 & 86.7 & 80 & 26.7 & 13.3 & 86.7 & 66.7 & 13.3 & 6.7 \\
HEA-1 & 4 & 100 & 100 & 75 & 75 & 100 & 100 & 50 & 25 \\
HEA-2 & 32 & 100 & 100 & 90.6 & 75 & 100 & 100 & 75 & 65.6 \\
Pregnancy & 22 & 100 & 100 & 86.4 & 68.2 & 100 & 100 & 31.8 & 13.6 \\
Overall & 79 & 97.3 & 89.5 & 62.4 & 49.6 & 97.3 & 83.3 & 34.0 & 22.2 \\
The passing percentage (\%) & of NNH (Niche-neutral hybrid) & model & \\
ABV & 6 & 0 & 0 & 0 & 0 & 81.4 & 57.9 & 19.9 & 14.1 \\
SBV & 15 & 6.7 & 6.7 & 6.7 & 6.7 & 74.0 & 55.2 & 23.9 & 14.8 \\
HEA-1 & 4 & 0 & 0 & 0 & 0 & 59.2 & 39.8 & 21.0 & 10.6 \\
HEA-2 & 32 & 53.1 & 15.6 & 3.1 & 3.1 & 78.8 & 68.1 & 32.5 & 19.8 \\
Pregnancy & 18 & 27.8 & 16.7 & 16.7 & 5.6 & 44.9 & 35.6 & 22.2 & 9.3 \\
Overall & 75 & 17.52 & 7.8 & 5.3 & 3.08 & 67.6 & 51.3 & 23.9 & 13.7
\end{tabular}

${ }^{*} N$ is the number of meta-communities (the number of individual subjects) investigated in each dataset.

(the left) and NNH (the right) models; for each model, the passing rate at metacommunity level and local community level was listed separately. Note that in Table 4, the passing percentages for MSN/NNH corresponding to a series of $P$-value thresholds were presented, but here we only explain the result from the traditional "default" threshold $(P=0.05)$ and the results for other threshold values are explained in the following discussion section.

First, regarding the overall performance of the MSN model, $97.3 \%$ of meta-communities and local communities passed the neutrality test, respectively. The range of neutrality percentage was $86.7-100 \%$ across five datasets. Therefore, the stochastic neutral forces seem to play a dominant role in shaping the assembly of HVMs. At a local community level, the performance of $\mathrm{NNH}$ is significantly lower than that of MSN, with local neutrality passing the neutrality test at rate of $67.6 \%$. However, at the metacommunity level, $\mathrm{NNH}$ also exhibited a moderate $17.5 \%$ of passing rate. Overall, niche differentiations appear to be moderate in the HVMs. In summary, the above findings indicate that both neutral and niche forces are in effect in shaping the community dynamics in the HVMs, but the neutral effects seem to play a dominant role.

\section{The Effects of BV on the Neutral-Niche Continuum in the HVM}

We further investigated the influence of BV (bacterial vaginosis) including both SBV (symptomatic BV) and ABV (asymptomatic $\mathrm{BV}$ ) on the balance between neutral and niche forces in shaping the HVM dynamics by performing Fisher's exact test and Student's $t$-test. The Fisher's exact test was performed to evaluate the effect of BV on the rate of passing the neutrality test (MSN) or testing the niche-neutral hybrid effect $(\mathrm{NNH})$ at the metacommunity level (the left side in Table 5), and Student's $t$-test on the passing rate of neutrality test at the local community level with either MSN or NNH model (the right side in Table 5). Similar to the previous sub-section, here we only

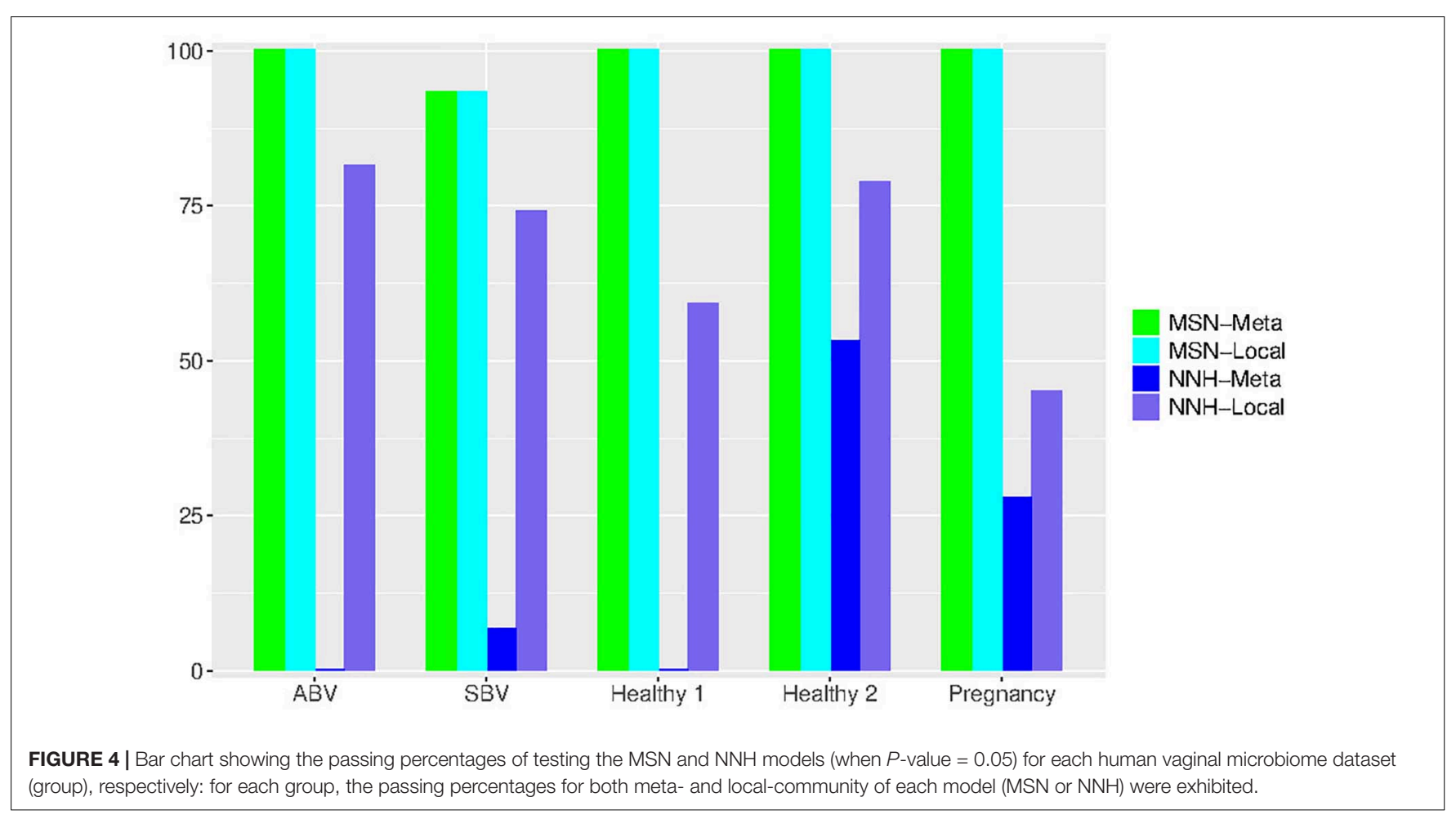


TABLE 5 | The $P$-values from testing the difference between various groups (ABV, SBV, HEA-1, HEA-2, and HEA) in their passing rates (from testing the MSN/NNH models) with Fisher exact test for the meta-community or Student's $t$-test for the local community $\left({ }^{\#, *}\right)$.

\begin{tabular}{|c|c|c|c|c|c|c|}
\hline \multirow[t]{2}{*}{ Models } & \multicolumn{3}{|c|}{$P$-value from Metacommunity (Fisher Exact Test) } & \multicolumn{3}{|c|}{$P$-value from Local community (Student's $t$-Test) } \\
\hline & Treatment & ${ }^{\#} P_{t}=0.05$ & ${ }^{\#} P_{t}=0.95$ & Treatment & ${ }^{\#} P_{t}=0.05$ & ${ }^{\#} P_{t}=0.95$ \\
\hline \multirow[t]{9}{*}{ MSN } & ABV vs. HEA-1 & 1.000 & 0.559 & ABV vs. HEA-1 & 1.000 & 1.000 \\
\hline & ABV vs. HEA-2 & 1.000 & 0.228 & ABV vs. HEA-2 & 1.000 & 1.000 \\
\hline & SBV vs. HEA-1 & 1.000 & 0.127 & SBV vs. HEA-1 & 1.000 & 1.000 \\
\hline & SBV vs. HEA-2 & 1.000 & 0.021 & SBV vs. HEA-2 & 1.000 & 1.000 \\
\hline & ABV vs. SBV & 1.000 & 1.000 & ABV vs. SBV & 1.000 & 1.000 \\
\hline & ABV vs. HEA (HEA-1++HEA-2) & 1.000 & 0.230 & ABV vs. HEA (HEA-1+HEA-2) & 1.000 & 1.000 \\
\hline & SBV vs. HEA (HEA-1+HEA-2) & 1.000 & 0.022 & SBV vs. HEA (HEA-1+HEA-2) & 1.000 & 1.000 \\
\hline & BV vs. HEA (HEA-1+HEA-2) & 1.000 & 0.011 & BV vs. HEA (HEA-1+HEA-2) & 1.000 & 1.000 \\
\hline & HEA-1 vs. HEA-2 & 1.000 & 1.000 & HEA-1 vs. HEA-2 & 1.000 & 1.000 \\
\hline \multirow[t]{9}{*}{ NNH } & ABV vs. HEA-1 & 1.000 & 1.000 & ABV vs. HEA-1 & 0.017 & 0.476 \\
\hline & ABV vs. HEA-2 & 1.000 & 1.000 & ABV vs. HEA-2 & 0.000 & 0.280 \\
\hline & SBV vs. HEA-1 & 1.000 & 1.000 & SBV vs. HEA-1 & 0.299 & 0.548 \\
\hline & SBV vs. HEA-2 & 1.000 & 1.000 & SBV vs. HEA-2 & 0.000 & 0.105 \\
\hline & ABV vs. SBV & 1.000 & 1.000 & ABV vs. SBV & 0.044 & 0.677 \\
\hline & ABV vs. HEA (HEA-1+HEA-2) & 1.000 & 1.000 & ABV vs. HEA (HEA-1+HEA-2) & 0.000 & 0.408 \\
\hline & SBV vs. HEA (HEA-1+HEA-2) & 0.517 & 1.000 & SBV vs. HEA (HEA-1+HEA-2) & 0.000 & 0.182 \\
\hline & BV vs. HEA (HEA-1+HEA-2) & 1.000 & 1.000 & BV vs. HEA (HEA-1+HEA-2) & 0.000 & 0.145 \\
\hline & HEA-1 vs. HEA-2 & 1.000 & 1.000 & HEA-1 vs. HEA-2 & 0.017 & 0.113 \\
\hline
\end{tabular}

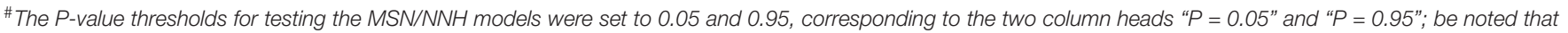

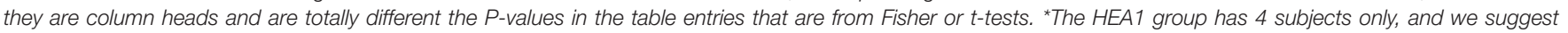

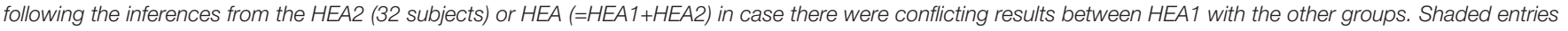
are comparisons with significant differences $(P \leq 0.05)$.

analyze the BV effects under traditional $P$-value $=0.05$ threshold and delay the analyses under alternative $P$-value thresholds to the discussion section.

Interestingly, both MSN and NNH exhibited slightly different results regarding the effects of $\mathrm{BV}$ status on the passing percentages of model tests. At the local community level, there appears to be significant differences in BV (SBV) and HEA (healthy groups) $(P$-value $<0.05$, Table 5$)$ according to the $\mathrm{NNH}$ model. However, at the metacommunity level, regardless of the MSN or NNH, the differences between various groups were statistically insignificant. The lack of difference between the HEA and pregnancy groups is also expected. Romero et al. (2014) defined a normal pregnancy as a woman with no obstetrical, medical or surgical complications, and delivered at term (38 to 42 weeks) without complications. The pregnancy group studied by Romero consisted of 22 normal pregnancies. Therefore, it appears that no statistically significant differences were detected between various groups in terms of MSN or NNH testing except for $\mathrm{NNH}$ at local community scale.

\section{DISCUSSION}

With traditional neutral theory of biodiversity, the spatially explicit or implicit model describing the metacommunity consisting of multiple local communities is the most frequently used metacommunity model for testing neutrality (Hubbell, 2001; Rosindell et al., 2011, 2012; Ma, 2020). The use of longitudinal community/meta-community samples to perform dynamic analysis of the niches vs. neutrality and to further assess and interpret the community static and dynamic properties by harnessing the neutral theory has been few but can be equally effective (Kalyuzhny et al., 2014a,b, 2015). The integrated modeling with $\mathrm{MSN} / \mathrm{NNH}$ in previous sections demonstrated another approach to generalizing the neutral-theoretic analysis to temporal meta-communities. Furthermore, we take advantages of a recent advance in computational statistics made by Harris et al. (2017) HDP-MSN machine learning algorithm. The HDP-MSN overcomes a significant computational bottleneck that existed in estimating the migration rates $(m)$ when the number of local communities is large, which prevented largescale testing of the UNTB with truly multi-site datasets. Nevertheless, truly multi-site datasets are scarce, especially in the studies of the human microbiome, where community samples are usually taken from unrelated individuals, and therefore dispersal (migration) among individuals is unlikely to occur on ecological timescales. In this study, we use the time-series sampling data in place of spatial sampling data. That is, the vaginal microbial community of each subject was sampled in patients at varying numbers of time points (6-60, see Table 1 ). By using time-series data with the MSN and NNH models, one can effectively evaluate the levels of stochastic neutral forces and deterministic niche forces in driving the community dynamics. In the case of time-series data, stochastic neutral forces may include stochastic fluctuations in demography (in the birth-death processes of bacterial cell divisions and deaths), which is the analog to ecological drift in neutral theory. The deterministic forces in time series data can include diversity- or 
TABLE 6 | Comparative summary of the performances of MSN and NNH models fitted to the human vaginal microbiome datasets of 79 subjects (meta-communities), summarized from Supplementary Tables 1, 2, under different $P_{t}$-value thresholds for testing MSN/MMH models.

\begin{tabular}{|c|c|c|c|c|c|c|c|c|c|}
\hline \multirow[t]{2}{*}{ Microbiome } & \multirow[t]{2}{*}{ Meta-Community } & \multicolumn{2}{|c|}{ MSN only } & \multicolumn{2}{|c|}{ NNH only } & \multicolumn{2}{|c|}{ Both MSN \& NNH } & \multicolumn{2}{|c|}{ NOT (MSN, NNH) } \\
\hline & & $N$ & $\%$ & $N$ & $\%$ & $N$ & $\%$ & $N$ & $\%$ \\
\hline \multicolumn{10}{|l|}{$P_{t}=0.05$} \\
\hline ABV & 6 & 6 & 100.0 & 0 & 0.0 & 0 & 0.0 & 0 & 0.0 \\
\hline SBV & 15 & 12 & 80.0 & 0 & 0.0 & 1 & 6.7 & 2 & 13.3 \\
\hline HEA-1 & 4 & 4 & 100.0 & 0 & 0.0 & 0 & 0.0 & 0 & 0.0 \\
\hline HEA-2 (32-Cohort) & 32 & 15 & 46.9 & 0 & 0.0 & 17 & 53.1 & 0 & 0.0 \\
\hline Pregnancy & 22 & 17 & 77.3 & 0 & 0.0 & 5 & 22.7 & 0 & 0.0 \\
\hline Overall & 79 & 54 & 68.4 & 0 & 0.0 & 23 & 29.1 & 2 & 2.5 \\
\hline \multicolumn{10}{|l|}{$P_{t}=0.5$} \\
\hline ABV & 6 & 4 & 66.7 & 0 & 0.0 & 0 & 0.0 & 2 & 33.3 \\
\hline SBV & 15 & 11 & 73.3 & 0 & 0.0 & 1 & 6.7 & 3 & 20.0 \\
\hline HEA-1 & 4 & 4 & 100.0 & 0 & 0.0 & 0 & 0.0 & 0 & 0.0 \\
\hline HEA-2 (32-Cohort) & 32 & 27 & 84.4 & 0 & 0.0 & 5 & 15.6 & 0 & 0.0 \\
\hline Pregnancy & 22 & 19 & 86.4 & 0 & 0.0 & 3 & 13.6 & 0 & 0.0 \\
\hline Overall & 79 & 65 & 82.3 & 0 & 0.0 & 9 & 11.4 & 5 & 6.3 \\
\hline \multicolumn{10}{|l|}{$P_{t}=0.9$} \\
\hline $\mathrm{ABV}$ & 6 & 2 & 33.3 & 0 & 0.0 & 0 & 0.0 & 4 & 66.7 \\
\hline SBV & 15 & 4 & 26.7 & 1 & 6.7 & 0 & 0.0 & 10 & 66.7 \\
\hline HEA-1 & 4 & 3 & 75.0 & 0 & 0.0 & 0 & 0.0 & 1 & 25.0 \\
\hline HEA-2 (32-Cohort) & 32 & 28 & 87.5 & 0 & 0.0 & 1 & 3.1 & 3 & 9.4 \\
\hline Pregnancy & 22 & 17 & 77.3 & 1 & 4.5 & 2 & 9.1 & 2 & 9.1 \\
\hline Overall & 79 & 54 & 68.4 & 2 & 2.5 & 3 & 3.8 & 20 & 25.3 \\
\hline \multicolumn{10}{|l|}{$P_{t}=0.95$} \\
\hline ABV & 6 & 1 & 16.7 & 0 & 0.0 & 0 & 0.0 & 5 & 83.3 \\
\hline SBV & 15 & 2 & 13.3 & 1 & 6.7 & 0 & 0.0 & 12 & 80.0 \\
\hline HEA-1 & 4 & 3 & 75.0 & 0 & 0.0 & 0 & 0.0 & 1 & 25.0 \\
\hline HEA-2 (32-Cohort) & 32 & 24 & 75.0 & 1 & 3.1 & 0 & 0.0 & 7 & 21.9 \\
\hline Pregnancy & 22 & 14 & 63.6 & 0 & 0.0 & 1 & 4.5 & 7 & 31.8 \\
\hline Overall & 79 & 44 & 55.7 & 2 & 2.5 & 1 & 1.3 & 32 & 40.5 \\
\hline
\end{tabular}

dominance-dependent regulatory forces for community stability (dynamics) (Ma and Ellison, 2018, 2019).

As explained in the previous section of results, the results from the integrated niche-neutral hybrid analyses under a default $P$-value $=0.05$ with MSN and NNH models in this study seem to suggest that neutral drifts play a dominant role in driving the community dynamics of the HVM, while the deterministic niche differentiation is moderate (approximately $17 \%$ in terms of passing NNH test). As further elaborated below, the assessment of the relative significance of neutral vs. niche may be strongly influenced by the model-choice (MSN or NNH) and $P$-value thresholds. It should also be reiterated that the conclusions obtained from this study are from analyzing the temporal dynamics data of the HVM, rather than from analyzing spatial metacommunity samples as usually performed in community ecology. In other words, the local communities in our analyses are simply the snapshots of an individual woman's vaginal microbiome dynamics. Therefore, niche differentiations are also "temporal differentiations," which might be relatively weak due to the nature of longitudinal observations. Future studies with "orthodox" spatial metacommunity samples should shed a more comprehensive picture on the community assembly and diversity maintenance of the HVMs. In the remainder of this article, we discuss two uncertainties regarding the test of the neutral and/or niche-neutral hybrid models.

First, it is well known that a significant challenge in investigating the mechanisms of community assembly or distinguishing the neutral from niche effects is that multiple independents models with possibly different ecological assumptions about the mechanisms may produce similar goodness-of-fittings to the same datasets, which is termed the lack of full model specificity in previous sections. This can make the inferences of definite mechanisms from different models difficult since the mapping from assumptions to mechanisms may not be one-to-one. Table 6 listed the breakups of successful fittings of the MSN/NNH models with $P$-value thresholds of $0.05-0.95$, classified as four groups including successfully fitted to "MSN-only," "NNH-only," "both MSN \& NNH," and "neither MSN nor NNH." Here, we first discuss the breakups when $P$-value threshold for testing the MSN/NNH model is set to 0.05 , and the other thresholds are discussed shortly below. As exhibited in Table 6, overall, the "MSN-only group" (fitted to MSN uniquely) takes about 68\% (ranged between 47 and 100\%) of all cases and "both MSN \& NNH" group (non-unique fittings) 


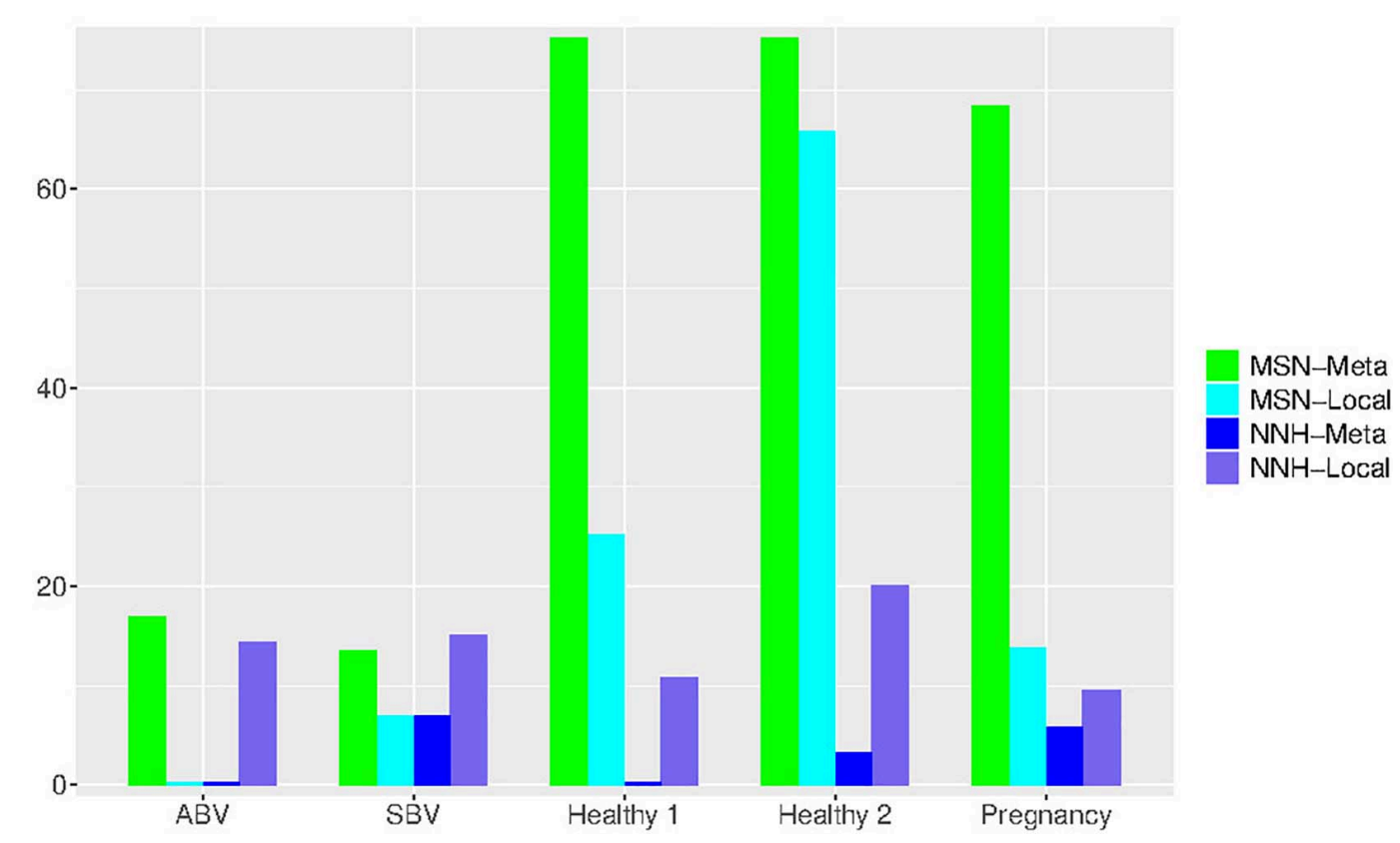

FIGURE 5 | Bar chart showing the passing percentages of testing the MSN and NNH models (when $P$-value $=0.95$ ) for each human vaginal microbiome dataset (group), respectively: for each group, the passing percentages for both meta- and local-community of each model (MSN or NNH) were exhibited.

takes about 29\%, (ranged between 0 and 53\%) and in less than $3 \%$ cases ( 2 out of 79 individuals) neither MSN nor NNH model was fitted successfully. Therefore, in the majority (68\% or 54 out of 79 individuals), the MSN model was able to uniquely interpret the neutral dynamics of the HVMs, when the $P$-value threshold for testing MSN/NNH was set to 0.05 .

Second, another dilemma that may lead to uncertainty in testing the neutral or niche-neutral hybrid models is the choice of $P$-value threshold. Traditionally, the $P$-value was set to 0.05 in testing the neutral theory; when $P>0.05$, the null hypothesis or model (satisfying the MSN or NNH model) cannot be rejected. In other words, when $P>0.05$, the observed community is considered indistinguishable from what the theoretical model predicts. In previous sections, $P$-value $=0.05$ was termed traditional "default." However, one may set $P$-value to other threshold values. The higher the $P$-value is, the more likely (the higher likelihood) that the community is consistent with the model prediction. That is, when the $P$-value is set to higher threshold values, it is more difficult to reject the null model. In terms of the neutrality test based on the MSN model, it implies that accepting neutral hypothesis is more reliable (conservative). In terms of the $\mathrm{NNH}$ model, it implies that accepting nonneutrality (niche differentiation) is more reliable (conservative). Consequently, when larger $P$-value thresholds are adopted, the confidence (reliability) to accepting the null model (MSN or $\mathrm{NNH}$ ) is raised and the confidence to reject the null model (MSM or NNH) is lowered. Table 4 listed the passing percentages (strictly, should be stated as percentage indistinguishable from model prediction) from testing MSN/NNH when $P$-value was set to $0.05,0.5,0.9$, and 0.95 , respectively. Obviously, as shown in Table 4, higher $P$-values correspond to a lower passing percentage of MSN-neutrality tests. When the $P$-value threshold was raised to 0.95 , the passing percentage of MSN-neutrality test declined to approximately $50 \%$, while the percentage was $97.3 \%$ when the $P$-value $=0.05$. Raising the $P$-value threshold from 0.05 to 0.95 is a rather dramatic increase of the confidence level for not rejecting the null neutral model (or accepting the null model), still nearly half the metacommunities (microbiomes of individuals) passed the MSN model, suggesting that the neutral drifts indeed play a significant role in shaping the dynamics of the HVM.

An interesting observation is that, when the $P$-value threshold was set to default 0.05 (Table 4), the passing percentages of MSM testing were not significantly different between different treatments (see Table 5 for Fisher's exact test). However, when the $P$-value threshold was set to 0.95 (Figure 5), the differences between the BV group (including $\mathrm{ABV}$ and SBV) and healthy groups (HEA1, HEA2, and pregnancy) were significant (13.3$16.7 \%$ vs. $68.2-75 \%)$. The healthy groups exhibited significantly more neutral communities than BV groups (Table 5). This result is actually puzzling. A traditional view has been that the vaginal microbiomes associated with BV usually have higher community diversity than the healthy counterparts, possibly due to the loss of dominant species such as Lactobacillus (e.g., Ma et al., 2012). Since communities with dominant species tend to contain more asymmetric interactions, they could be less likely neutral. Therefore, this puzzling result appears to contradict with the traditional view. Of course, the relationship between dominant species and non-neutrality is not necessarily positive; we hope that future more mechanistic studies will resolve the apparent inconsistency.

The adoption of different $P$-value thresholds may also affect the previously discussed specificity (uniqueness) of MSN/NNH 
model fittings. Table 6 shows the breakups of four categories (MSN-only, NNH-only, both MSN \& NNH, neither MSN nor $\mathrm{NNH}$ ) under different $P$-values ranging from $P$-value $=0.05$ to 0.95 . It appears that the model specificity of MSN seems to increase with the increase of $P$-value threshold adopted. This result should be expected since the increased $P$-value should raise the confidence level for accepting the null (MSN or NNH) model, and the passing percentages judged with higher confidence would decline accordingly.

Finally, the two previously discussed uncertainties associated with testing the neutral or niche-neutral hybrid models can be interdependent and proper resolving them requires both ecological science and statistical art. There may not be a perfect solution for resolving those issues due to both the ecological complexity and the limitation of frequentist statistical approaches. The art lies in balancing the trade-off between reliability (confidence) in hypothesis testing and model specificity. It is hoped that the demonstrative analysis and discussion included in this study with the HVMs will also be useful for other ecological and evolutionary modeling of biodiversity and biogeography.

\section{DATA AVAILABILITY STATEMENT}

The original contributions presented in the study are included in the article/Supplementary Material, further inquiries can be directed to the corresponding author.

\section{AUTHOR CONTRIBUTIONS}

ZM designed and performed the study and wrote the manuscript.

\section{REFERENCES}

Alonso, D., Etienne, R., and Mckane, A. (2006). The merits of neutral theory. Trends Ecol. Evol. 21, 451-457. doi: 10.1016/j.tree.2006.03.019

Antoniak, C. E. (1974). Mixtures of dirichlet processes with applications to bayesian nonparametric problems. Ann. Statist. 2, 1152-1174.

Chisholm, R. A., and Pacala, S. W. (2010). Niche and neutral models predict asymptotically equivalent species abundance distributions in high-diversity ecological communities. Proc. Natl Acad. Sci. U S A. 107, 15821-15825. doi: 10.1073/pnas.1009387107

Darwin, C. (1859). On the Origin of Spices. London: John Murray.

Etienne, R. S. (2007). A neutral sampling formula for multiple samples and an 'exact' test of neutrality. Ecol. Lett. 10, 608-618. doi: 10.1111/j.1461-0248.2007. 01052.x

Etienne, R. S. (2009a). Improved estimation of neutral model parameters for multiple samples with different degrees of dispersal limitation. Ecology 90, 847-852. doi: $10.1890 / 08-0750.1$

Etienne, R. S. (2009b). Maximum likelihood estimation of neutral model parameters for multiple samples with different degrees of dispersal limitation. J. Theor. Biol. 257, 510-514. doi: 10.1016/j.jtbi.2008.12.016

Fisher, C. K., and Mehta, P. (2014). The transition between the niche and neutral regimes in ecology. Proc. Natl. Acad. Sci. U S A. 111, 13111-13116. doi: 10. 1073/pnas. 1405637111

Fredricks, D. N. (2011). Molecular methods to describe the spectrum and dynamics of the vaginal microbiota. Anaerobe 17, 191-195. doi: 10.1016/j.anaerobe.2011. 01.001

\section{FUNDING}

This study received funding from the following sources: A National Natural Science Foundation (NSFC) Grant (No. 31970116) on "Medical Ecology of Human Microbiome", and the "Cloud-Ridge Industry Technology Leader" Award. An International Cooperation Grant (YNST) on Genomics and Metagenomics Big Data. The funders played no roles in interpreting the results.

\section{ACKNOWLEDGMENTS}

I deeply appreciate Hubbell for reviewing this manuscript and for his insightful comments and suggestions to improve my work. I am obliged to express my sincere appreciation to Rampal Etienne for his insightful advice and comments on testing neutral models based on the $P$-value. I thank Christopher Quince for his professional cooperation and support in updating their published work. I appreciate the computational support from LW Li of the Chinese Academy of Sciences.

\section{SUPPLEMENTARY MATERIAL}

The Supplementary Material for this article can be found online at: https://www.frontiersin.org/articles/10.3389/fmicb. 2021.699939/full\#supplementary-material

Supplementary Table 1 | Test results of fitting the MSN (multi-site neutral) model to the vaginal microbiome datasets.

Supplementary Table 2 | Fitting the NNH (Niche-Neutral Hybrid) model to the vaginal microbiome datasets.

Fredricks, D. N., Fiedler, T. L., and Marrazzo, J. M. (2005). Molecular identification of bacteria associated with bacterial vaginosis. N. Engl. J. Med. 353, 1899-1911. doi: $10.1056 /$ nejmoa 043802

Gajer, P., Brotman, R. M., Bai, G., Sakamoto, J., Schütte, U. M., Zhong, X., et al. (2012). Temporal dynamics of the human vaginal microbiota. Sci. Trans. Med. 132:132ra52.

Gilbert, J. A., Blaser, M. J., Caporaso, J. G., Jansson, J. K., Lynch, S. V., and Knight, R. (2018). Current understanding of the human microbiome. Nat. Med. 24, 392-400. doi: $10.1038 / \mathrm{nm} .4517$

Grinnell, J. (1917). The niche-relationships of the California Thrasher. Auk 34, 427-433. doi: 10.2307/4072271

Harris, K., Parsons, T. L., Ijaz, U. Z., Lahti, L., Holmes, I., Quince, C., et al. (2017). Linking statistical and ecological theory: hubbell's unified neutral theory of biodiversity as a hierarchical dirichlet process. Proc. IEEE 105, 516-529. doi: $10.1109 /$ jproc.2015.2428213

Holt, R. D. (2009). Bringing the hutchinsonian niche into the 21st century: ecological and evolutionary perspectives. Proc. Natl. Acad. Sci. U S A. 106, 19659-19665. doi: 10.1073/pnas.0905137106

Hubbell, S. P. (2001). The Unified Neutral Theory of Biodiversity and Biogeography. Princeton, NJ: Princeton University Press.

Hubbell, S. P. (2006). Neutral theory and the evolution of ecological equivalence. Ecology 87, 1387-1398. doi: 10.1890/0012-9658(2006)87[1387:ntateo]2.0.co;2

Hutchinson, G. E. (1957). Concluding remarks. Cold Spring Harb. Symp. 22, $415-427$.

Jeraldo, P., Sipos, M., Chia, N., Brulc, J. M., Dhillon, A. S., Konkel, M. E., et al. (2012). Quantification of the relative roles of niche and neutral processes in 
structuring gastrointestinal microbiomes. Proc. Natl. Acad. Sci. U S A. 109, 9692-9698. doi: 10.1073/pnas.1206721109

Kalyuzhny, M., Kadmon, R., and Shnerb, N. M. (2015). A neutral theory with environmental stochasticity explains static and dynamic properties of ecological communities. Ecol. Lett. 18, 572-580. doi: 10.1111/ele.12439

Kalyuzhny, M., Kadmon, R., and Shnerb, N. M. (2014a). A generalized neutral theory explains static and dynamic properties of biotic communities. Quan. Biol. 21, 62-74.

Kalyuzhny, M., Seri, E., Chocron, R., Flather, C. H., Kadmon, R., and Shnerb, N. M. (2014b). Niche versus neutrality: a dynamical analysis. Am. Nat. 184, 439-446. doi: $10.1086 / 677930$

Knight, R., Callewaert, C., Marotz, C., Hyde, E. R., Debelius, J. W., McDonald, D., et al. (2017). The microbiome and human biology. Ann. Rev. Genom. Hum. Genet. 18, 65-86.

Lynch, S. V., and Pedersen, O. (2016). The human intestinal microbiome in health and disease. N. Engl. J. Med. 375, 2369-2379. doi: 10.1056/NEJMra1600266

Ma, B., Forney, L. J., and Ravel, J. (2012). The vaginal microbiome: rethinking health and disease. Annu. Rev. Microbiol. 66, 371-389. doi: 10.1146/annurevmicro-092611-150157

Ma, Z. S. (2020). Niche-neutral theoretic approach to mechanisms underlying the biodiversity and biogeography of human microbiomes. Evol. Appl. 2020, 1-13. doi: 10.1111/eva.13116

Ma, Z. S., and Ellison, A. M. (2018). A unified concept of dominance applicable at both community and species scale. Ecosphere 9:e02477. doi: 10.1002/ecs2.2477

Ma, Z. S., and Ellison, A. M. (2019). Dominance network analysis provides a new framework for studying the diversity-stability relationship. Ecol. Monographs 89:e01358. doi: 10.1002/ecm.1358

Matthews, T. J., and Whittaker, R. J. (2014). Neutral theory and the species abundance distribution: recent developments and prospects for unifying niche and neutral perspectives. Ecol. Evol. 4, 2263-2277.

McGill, B. J., Maurer, B. A., and Weiser, M. D. (2006). Empirical evaluation of neutral theory. Ecology 87, 1411-1423. doi: 10.1890/0012-9658(2006)87[1411: eeont]2.0.co;2

Noble, A. E., and Fagan, W. F. (2015). A niche remedy for the dynamical problems of neutral theory. Theoret. Ecol. 8, 149-161. doi: 10.1007/s12080-014-0240-x

Ofiteru, I. D., Lunn, M., Curtis, T. P., Wells, G. F., and Criddle, C. S. (2010). Combined niche and neutral effects in a microbial wastewater treatment community. Proc. Natl. Acad. Sci. U S A. 107, 15345-15350. doi: 10.1073/pnas. 1000604107

Pigolotti, S., and Cencini, M. (2013). Species abundances and lifetimes: from neutral to niche-stabilized communities. J. Theor. Biol. 338, 1-8. doi: 10.1016/ j.jtbi.2013.08.024

Ravel, J., Brotman, R. M., Gajer, P., Ma, B., Nandy, M., Fadrosh, D. W., et al. (2013). Daily temporal dynamics of vaginal microbiota before, during and after episodes of bacterial vaginosis. Microbiome 1:29.

Romero, R., Hassan, S. S., Gajer, P., Tarca, A. L., Fadrosh, D. W., Nikita, L., et al. (2014). The composition and stability of the vaginal microbiota of normal pregnant women is different from that of non-pregnant women. Microbiome 2:4.

Rosindell, J., Hubbell, S. P., and Etienne, R. S. (2011). The unified neutral theory of biodiversity and biogeography at age ten. Trends Ecol. Evol. 26, 340-348. doi: $10.1016 /$ j.tree.2011.03.024
Rosindell, J., Hubbell, S. P., He, F., Harmon, L. J., and Etienne, R. S. (2012). The case for ecological neutral theory. Trends Ecol. Evol. 27, 203-208. doi: 10.1016/j.tree.2012.01.004

Sloan, W. T., Lunn, M., Woodcock, S., Head, I. M., Nee, S., and Curtis, T. P. (2006). Quantifying the roles of immigration and chance in shaping prokaryote community structure. Environ. Microbiol. 8, 732-740. doi: 10.1111/j.14622920.2005.00956.x

Sloan, W. T., Woodcock, S., Lunn, M., Head, I. M., and Curtis, T. P. (2007). Modeling taxa-abundance distributions in microbial communities using environmental sequence data. Microb. Ecol. 53, 443-455. doi: 10.1007/s00248006-9141-x

Sobel, J. D. (1999). Is there a protective role for vaginal flora? Curr. Infect. Dis. Rep. 1, 379-383. doi: 10.1007/s11908-999-0045-z

Stokes, C. J., and Archer, S. R. (2010). Niche differentiation and neutral theory: an integrated perspective on shrub assemblages in a parkland savanna. Ecology 91, 1152-1162. doi: 10.1890/08-1105.1

Tang, J., and Zhou, S. (2013). Hybrid niche-neutral models outperform an otherwise equivalent neutral model for fitting coral reef data. J. Theor. Biol. 317 212-218. doi: 10.1016/j.jtbi.2012.10.019

Teh, Y. W., Jordan, M., Beal, M., and Blei, D. (2006). Hierarchical dirichlet processes. J. Am. Statist. Assoc. 101, 1566-1581.

Tilman, D. (2004). Niche tradeoffs, neutrality, and community structure: a stochastic theory of resource competition, invasion, and community assembly. Proc. Natl. Acad. Sci. U S A. 101, 10854-10861. doi: 10.1073/pnas.0403458101

Volkov, I., Banavar, J. R., Hubbell, S. P., and Maritan, A. (2003). Neutral theory and relative species abundance in ecology. Nature 424, 1035-1037. doi: 10.1038/ nature 01883

Volkov, I., Banavar, J. R., Hubbell, S. P., and Maritan, A. (2007). Patterns of relative species abundance in rainforests and coral reefs. Nature 450, 45-49. doi: $10.1038 /$ nature 06197

Young, V. B. (2017). The role of the microbiome in human health and disease: an introduction for clinicians. BMJ 2017:356. doi: 10.1136/bmj. j831

Conflict of Interest: The author declares that the research was conducted in the absence of any commercial or financial relationships that could be construed as a potential conflict of interest.

Publisher's Note: All claims expressed in this article are solely those of the authors and do not necessarily represent those of their affiliated organizations, or those of the publisher, the editors and the reviewers. Any product that may be evaluated in this article, or claim that may be made by its manufacturer, is not guaranteed or endorsed by the publisher.

Copyright (C) $2021 \mathrm{Ma}$. This is an open-access article distributed under the terms of the Creative Commons Attribution License (CC BY). The use, distribution or reproduction in other forums is permitted, provided the original author(s) and the copyright owner(s) are credited and that the original publication in this journal is cited, in accordance with accepted academic practice. No use, distribution or reproduction is permitted which does not comply with these terms. 